\title{
61. Discharge Process in Geiger-Müller Counters. VI.
}

\author{
Time interval of "Nachentladung". \\ By Osamu Minakaw.A. \\ Institute of Physical and Chemical Research, Tokyo. \\ (Comm. by S. Nishikawa, M.I.A., May 12, 1944.)
}

The origin of "Nachentladung" is not yet completely cleared up, at present. Trost" thought it most plausible to explain his experimental results, by ascribing the origin of " $\mathrm{N}$. E."*) to the emission of photoelectrons from the wall by the photons which are emitted in the collision of positive and negative ions. The writer ${ }^{2)}$ has already introduced the following mechanism from the various experimental facts. When small and simple ions, produced in the counter discharge, come to the wall, they pull out electrons from the wall through the exceedingly thin surface layer of non-conductor and the recombination takes place, a rery small part of such electrons, however, may escape into the counter volume and it may be thought that this should be the origin of "Nachentladung". Simoda and Ogawa ${ }^{3)}$ have emphasized from their experiments with counters with grids that the origin of a part of " N.E." is due to photons which are produced in the discharge and the larger part of it may be ascriber to the positive ions, which produce electrons in the gas near the counter wall where the electric field is week.

To assume negative ions, however, for the delay of excitation may be excluded because of the following reasons.

1. Efficiency of the counter examined by the coincidence method is almost $100 \%$.

2. In comparing the probability of occurrence of "N. E." for the counters containing hydrogen (electro-positive gas) as impurity with those containing oxygen (electro-negative gas), the former has rather large probability of "N. E."

3. The probability of inducing discharge is not proportional to that of "N. E."')

4. Liberation of electrons from negative ions even in the region of the strong electric field as in the counter may be highly improbable.

The definite experimental evidence to exclude photons completely from the cause of "Nachentladung" is not at present exist, and photons are undoubtedly convenient to explain the experimental results about the behavior of "N. E." obtained with counters with grids. It is, however, not impossible to explain all the results by ascribing the origin of "N. E." only to positive ions.

1) A. Trost: Zeits. f. Phys., 105 (1937), 399.

2) (O. Ninakawa: Proc. 18 (1942), 644, 19 (1943), 19.

3) K. Simoda and I. Ogawa: Nippon Sugaku-Buturigaku Kaisi, 2 (2603), 241.

1) (). Minakima: Proc. 18 (1942), 468.

) I'his is an abbreviation of "Nachentladung". 
At any rate, for the origin of "N.E." the positive ions comes into question. Simoda and Ogawa has drawn their conclusion, mentioned above, from the comparison of the time of occurrence of "N. E." with the position of mean positive ion sheath. This time relation, however, is a matter of course from the standpoint of the above mentioned mechanism of small positive ions. To assume that positive ions emit electrons from tne gas molecules near the counter wall, cannot explain the evident fact that the probability of "Nachentladung" is different in different wall materials.
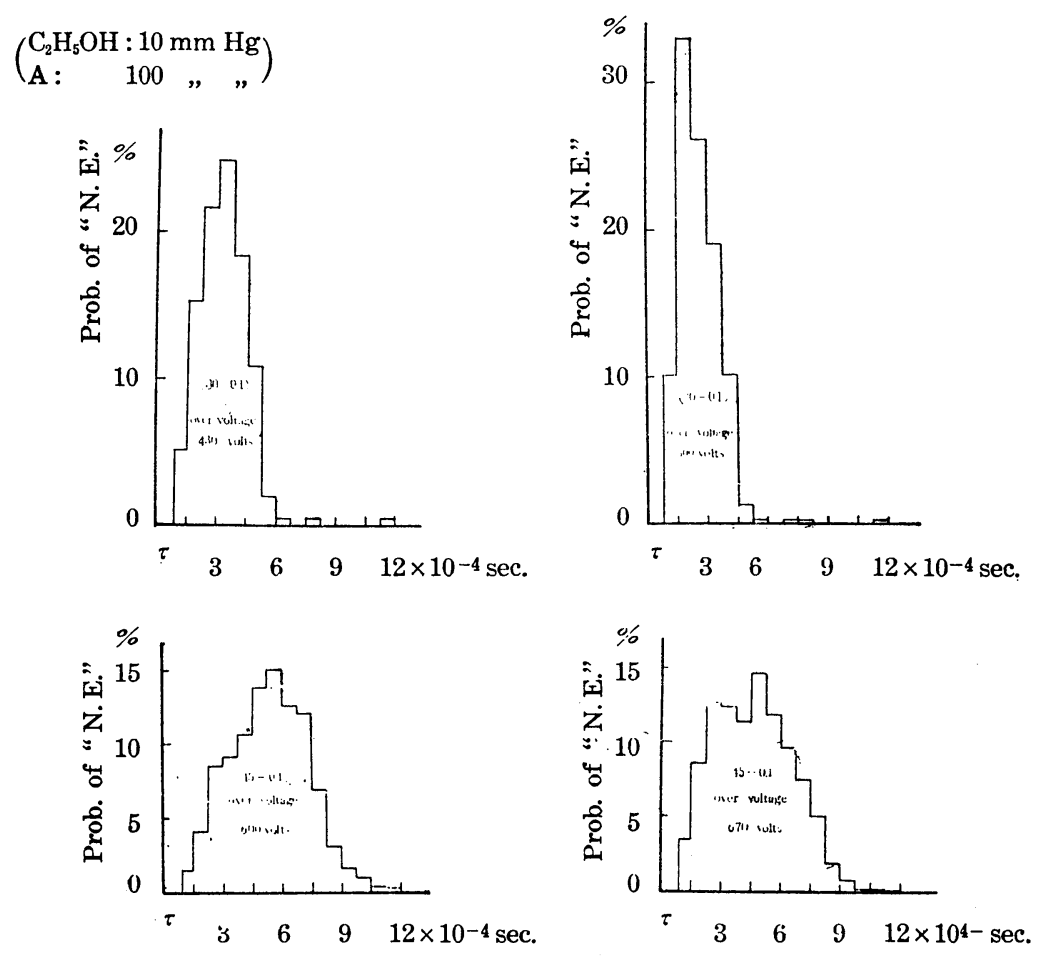

Fig. 1.

Under such circumstances, in order to test further the dipole mechanism at the cathode surface, the time distribution of "Nachentladung" was examined. Results are shown in Figs. 1-3. Fig. 1 shows the variation with over voltage and with diameters of the cathode wall. It is clear that "N. E." is distributed in a certain time intervals, the delay is shortened with the over voltage, and that the distribution is flattened when the diameter of the wall is large.

Fig. 2 shows the difference in distribution in different cathode materials. The difference in distribution with the same counter but with different impurity is shown in Fig. 3. In every case the ordinate represents the percentage of occurrence, i.e. the total area under the curve is put to be equal. 


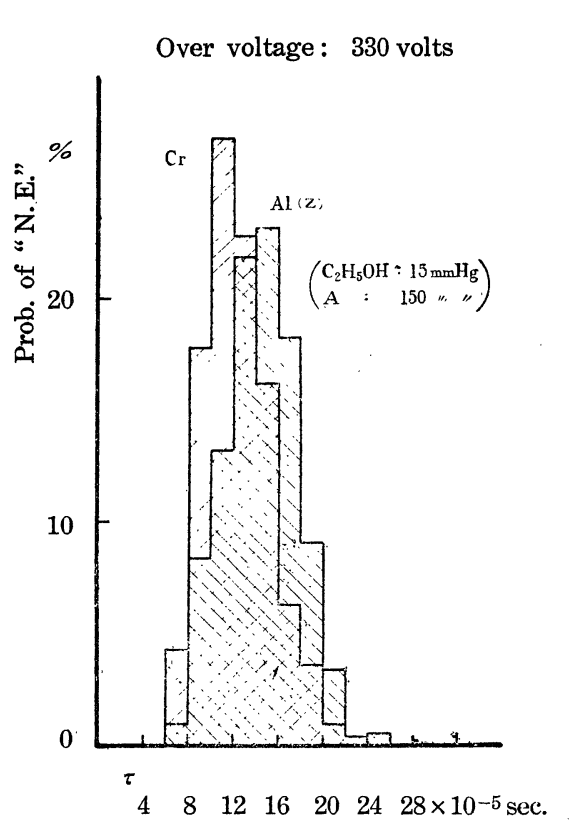

Fig. 2.

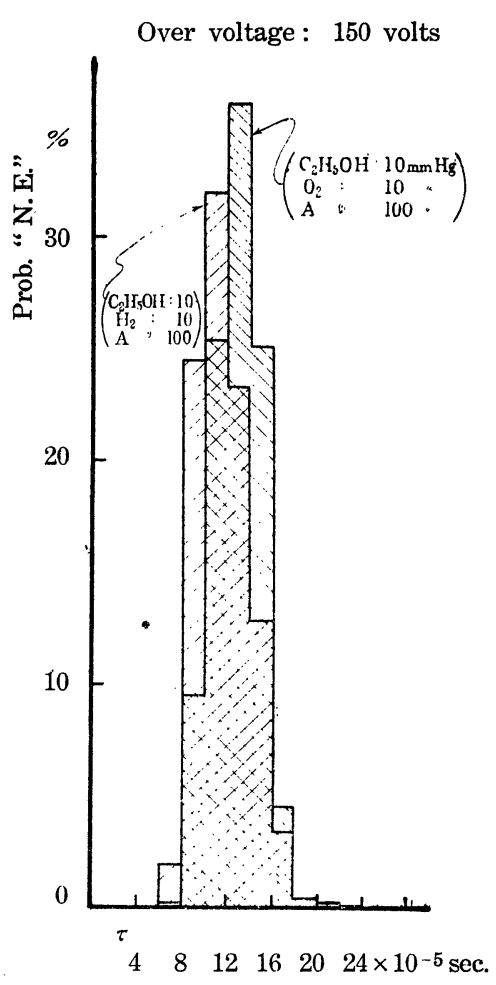

Fig. 3.

From these results it can also be confirmed that the "N.E." occurs when the positive ions reach the cathode.

In conclusion the vriter wishes to express his best thanks to Prof. H. Nagaoka and to Prof. S. Nishikawa for their kind interests and encouragement throughout this work. 